\title{
ON THE DYNAMICS OF A PERIODIC DELAY LOGISTIC EQUATION WITH DIFFUSION
}

\section{K. Gopalsamy, Pei-Xuan Weng}

Sufficient conditions are obtained for the existence of a globally attractive positive periodic solution of the periodic diffusive delay logistic system

$$
\begin{gathered}
\frac{\partial N(x, t)}{\partial t}=D \frac{\partial^{2} N(x, t)}{\partial x^{2}}+r(t) N(x, t)\left[1-\frac{N(x, t-n \tau)}{K(t)}\right] \\
\quad 0<x<\ell, \quad t>0 \\
\frac{\partial N(0, t)}{\partial x}=\frac{\partial N(\ell, t)}{\partial x}=0, \quad t \geqslant-n \tau
\end{gathered}
$$

in which $r$ and $K$ are positive periodic functions of period $\tau, n$ is a positive integer and $D$ is a nonnegative number; sufficient conditions are also obtained for all positive solutions to be oscillatory about the periodic solution.

\section{INTRODUCTION}

In a recent article, Zhang and Gopalsamy [34] have studied the periodic delay logistic equation

$$
\frac{d x(t)}{d t}=r(t) x(t)\left[1-\frac{x(t-n \tau)}{K(t)}\right]
$$

where $r, K$ are positive continuous periodic functions of period $r \geqslant 0$ and $n$ is a positive integer. In the following we are concerned with an investigation of the asymptotic and oscillatory behaviour of the solutions of the initial boundary value problem

$$
\begin{aligned}
& \frac{\partial N(x, t)}{\partial t}=D \frac{\partial^{2} N(x, t)}{\partial x^{2}}+r(t) N(x, t)\left[1-\frac{N(x, t-n \tau)}{K(t)}\right] \\
& x \in(0, \ell), \quad t>0 ; \\
& \frac{\partial N(0, t)}{\partial x}=\frac{\partial N(\ell, t)}{\partial x}=0, \quad t \geqslant-n \tau ; \\
& N(x, s)=\phi(x, s)>0, \quad x \in[0, \ell], \quad s \in[-n \tau, 0]
\end{aligned}
$$

Received 11 February 1991

Copyright Clearance Centre, Inc. Serial-fee code: 0004-9729/92 \$A2.00+0.00. 
in which $\phi$ is a positive function assumed to be sufficiently smooth to guarantee the existence of classical solutions of the boundary value problem (1.2) $-(1.3)$. When $\tau=0$, the equation (1.2) becomes an autonomous equation which has been known as Fisher's equation and there exists an extensive literature on such an autonomous equation. When the diffusion term is absent in (1.2) and $r$ and $K$ are positive constants, the equation (1.2) is also known as Hutchinson's [9] equation whose mathematical analysis can be found in the works of Wright [29], Kakutani and Markus [13] and Jones [10].

We note that autonomous equations of the type (1.2) - (1.3) have been discussed by several authors (Yoshida [31], Lin and Khan [18], Yoshida and Khisimoto [32], Yamada [30], Green and Stech [8], Cohen and Rosenblatt [4], Murray [22], Memory [20], Morita [21], Luckhaus [19], Redlinger [25], Tesei [27] and Schiaffino [26]). The results of these authors are not concerned with periodic solutions and mostly are concerned with the instability of the positive equilibrium of the autonomous equations such as (1.2) in which $r$ and $K$ are positive constants. Our purpose of study of (1.2) - (1.4) is to derive sufficient conditions for all solutions of $(1.2)-(1.4)$ to "converge" to the unique positive "periodic solution" whose existence will be established in the next section. The existence of periodic solutions of semilinear parabolic partial differential equations (with no time delays) has been usually established by fixed point methods (see for instance Amann [1] and Kolesov [14]). We refer to Vejvoda [28] for an extensive bibliography on time-periodic solutions of partial differential equations; we note that stability characteristics of these periodic solutions have scarcely been discussed in the existing literature. Our interest in the periodic parabolic system (1.2) - (1.3) has arisen from the potential applications of (1.2) - (1.3) in modelling the spatial spread of a population density in a temporally periodic environment.

In population dynamics, $N(x, t)$ of $(1.2)$ denotes the density of a population and so we will be interested only in the nonnegative solutions of (1.2)-(1.4). The homogeneous Neumann boundary conditions (1.3) mean that the species is confined to the linear habitat $[0, \ell]$ and cannot cross the boundaries to move to the outside of the habitat. It is not obvious that nontrivial solutions of (1.2) - (1.4) will remain nonnegative so long as they exist; we shall briefly discuss this aspect. Suppose for instance there exists a point $\left(x_{0}, t_{0}\right), t_{0} \geqslant 0, x_{0} \in[0, \ell]$ where $N\left(x_{0}, t_{0}\right)<0$. For $(x, t) \in \widetilde{E}=\{(x, t) \mid$ $\left.(x, t) \in[0, \ell] \times\left[0, t_{0}\right]\right\}$, we define $m_{\lambda}$ by the following:

$$
m_{\lambda}(x, t)=N(x, t) e^{-\lambda t}
$$

in which $\lambda>0$ will be chosen suitably. It follows from (1.2) that

$$
\frac{\partial m_{\lambda}(x, t)}{\partial t}=D \frac{\partial^{2} m_{\lambda}(x, t)}{\partial x^{2}}+m_{\lambda}(x, t)\left[r(t)-\lambda-r(t) \frac{N(x, t-\tau)}{K(t)}\right]
$$


Let

$$
\begin{gathered}
L=D \frac{\partial^{2}}{\partial x}-\frac{\partial}{\partial t} \\
h_{\lambda}(x, t)=r(t)-\lambda-r(t) \frac{N(x, t-\tau)}{K(t)} .
\end{gathered}
$$

Then (1.6) becomes

$$
L\left[m_{\lambda}\right]+h_{\lambda}(x, t) m_{\lambda}=0 .
$$

We can now choose $\lambda$ large enough so that

$$
h_{\lambda}(x, t)<0, \quad \text { for } \quad(x, t) \in \tilde{E} .
$$

Since $m_{\lambda}\left(x_{0}, t_{0}\right)<0$, by continuity of $m_{\lambda}$ on $\tilde{E}, m_{\lambda}$ must have a negative minimum in $\widetilde{E}$ at say $\left(x^{*}, t^{*}\right)$. Hence by the parabolic maximum principle [24, p.173], we know $\left(x^{*}, t^{*}\right) \notin E=\left\{(x, t) \mid 0<x<\ell, 0<t \leqslant t_{0}\right\}$ since otherwise,

$$
m_{\lambda}(x, t) \equiv \text { constant for }(x, t) \in[0, \ell] \times\left[0, t^{*}\right]
$$

which is impossible from (1.5) and (1.6). From the initial condition (1.4), we have

$$
\left(x^{*}, t^{*}\right) \notin E_{0} \text { where } E_{0}=\{(x, t) \mid 0<x<\ell, t=0\}
$$

hence $\left(x^{*}, t^{*}\right)$ must belong to the boundary $\Gamma$ of $E$ where

$$
\Gamma=\left\{(x, t) \mid 0 \leqslant t \leqslant t_{0}, \quad x=0, \quad x=\ell\right\}
$$

Let us suppose that $x^{*}=0$ and $m_{\lambda}\left(0, t^{*}\right)$ is a negative minimum of $m_{\lambda}$ on $\widetilde{E}$. But we have from the homogeneous Neumann boundary condition for $N$ that

$$
\frac{\partial m_{\lambda}\left(0, t^{*}\right)}{\partial x}=0
$$

The slope of the curve $m_{\lambda}\left(x, t^{*}\right)$ is nondecreasing at $\left(0, t^{*}\right)$ and the curvature of the curve $m_{\lambda}\left(x, t^{*}\right)$ is either concave upward or horizontal when $x \in[0, \delta)$ for some $\delta>0$ with $t^{*}$ fixed; as a consequence, for any $\varepsilon>0$ we can find a $\delta_{e}>0$ such that

$$
\frac{\partial^{2} m_{\lambda}\left(x, t^{*}\right)}{\partial x^{2}} \geqslant 0, \quad \frac{\partial m_{\lambda}\left(x, t^{*}\right)}{\partial t}<\varepsilon, \quad m_{\lambda}\left(x, t^{*}\right)<0 \text { for } x \in\left(0, \delta_{\varepsilon}\right) \text {. }
$$

We note that $x^{*}=0$ and $h_{\lambda}$ is negative on the closed set $\tilde{E}$ and hence $h_{\lambda}$ has a negative maximum on $\tilde{E}$. We choose a positive number $\varepsilon_{0}$ such that

$$
4 \varepsilon_{0}=m_{\lambda}\left(x^{*}, t^{*}\right) \max _{(x, t) \in \tilde{E}}\left\{r(t)-\lambda-r(t) \frac{N(x, t-n \tau)}{K(t)}\right\} .
$$


Then from the continuity of $m_{\lambda}$, there exists a $\delta_{0}>0$ such that

$$
\begin{aligned}
e_{\lambda}\left(x, t^{*}\right) & \left\{r\left(t^{*}\right)-\lambda-r\left(t^{*}\right) \frac{N\left(x, t^{*}-n \tau\right)}{K\left(t^{*}\right)}\right\} \\
& \geqslant m_{\lambda}\left(x, t^{*}\right) \max _{(x, t) \in \widetilde{E}}\left\{r(t)-\lambda-r(t) \frac{N(x, t-n \tau)}{K(t)}\right\} \\
& \geqslant 2 \varepsilon_{0}, \quad \text { for } x \in\left(0, \delta_{0}\right) .
\end{aligned}
$$

Let $\delta_{1}=\min \left\{\delta_{0}, \delta_{e_{0}}\right\}$. Then from (1.6) and (1.7), we derive

$$
\begin{aligned}
\varepsilon_{0} & >\frac{\partial m_{\lambda}\left(x, t^{*}\right)}{\partial t} \\
& =D \frac{\partial^{2} m_{\lambda}\left(x, t^{*}\right)}{\partial x^{2}}+m_{\lambda}\left(x, t^{*}\right)\left[r\left(t^{*}\right)-\lambda-r\left(t^{*}\right) \frac{N\left(x, t^{*}-n \tau\right)}{K\left(t^{*}\right)}\right] \\
& \geqslant m_{\lambda}\left(x, t^{*}\right)\left[r\left(t^{*}\right)-\lambda-r\left(t^{*}\right) \frac{N\left(x, t^{*}-n \tau\right)}{K\left(t^{*}\right)}\right] \\
& \geqslant 2 \varepsilon_{0}, \quad x \in\left(0, \delta_{1}\right) .
\end{aligned}
$$

This is impossible. Thus $m_{\lambda}$ cannot have a negative minimum at $x=0$. A similar analysis can be used to show that $m_{\lambda}$ cannot have a negative minimum at $x=\ell$. Thus $m_{\lambda}$ cannot have a negative minimum on $\widetilde{E}$ and this is valid for any positive $t_{0}$. Thus we conclude that $m_{\lambda} \geqslant 0$ for $x \in[0, \ell]$ and $t \geqslant-n \tau$. It now follows from (1.5) that it is impossible for $N(x, t)$ to become negative; thus

$$
N(x, t) \geqslant 0, \quad \text { for } \quad x \in[0, \ell], \quad t>0 .
$$

The existence of nonnegative solutions of (1.2) - (1.4) for all $t>0$ can be established by continuation using the method of steps and Green's functions (see for instance Britton [3]). We refer to Leung [17] and Okubo [23] for applications of partial differential equations to population biology especially population dynamics.

\section{Periodic SOlution and its global attractivity}

It has been noted by Zhang and Gopalsamy [34] that the periodic ordinary differential equation

$$
\frac{d y(t)}{d t}=r(t) y(t)\left[1-\frac{y(t)}{K(t)}\right]
$$

has a unique periodic solution $y^{*}$ of period $\tau$, where

$$
\begin{aligned}
y^{*}(t) & =\left[\int_{0}^{\infty} \frac{r(t-s)}{K(t-s)} \exp \left\{-\int_{0}^{s} r(t-\sigma) d \sigma\right\} d s\right]^{-1} \\
& =\frac{1-\exp [-\langle r\rangle]}{\int_{0}^{r} \frac{r(t-s)}{K(t-s)} \exp \left[-\int_{0}^{s} r(t-\sigma) d \sigma\right] d s}
\end{aligned}
$$


in which

$$
\langle r\rangle=\int_{0}^{r} r(s) d s
$$

The solution $y^{*}$ has been shown earlier by Coleman $[5,6]$ to be a global attractor of all positive solutions of the periodic system (2.1). The periodic solution $y^{*}$ of (2.1) is also found to be a periodic solution of the periodic parabolic boundary value problem (1.2) - (1.3). We are now interested in the derivation of sufficient conditions for all positive solutions of (1.2) - (1.4) to converge to the spatially homogeneous positive periodic solution $y^{*}$. We note that $y^{*}$ is a temporally nonstationary and spatially homogeneous equilibrium solution of (1.2) - (1.4) and our result below (Theorem 2.3) provides sufficient conditions for the global asymptotic stability of this nonstationary equilibrium. Our technique is based on a comparison method. The next result is preparatory and provides a useful comparison of solutions of $(1.2)-(1.3)$ with those of a lumped parameter system.

Theorem 2.1. Let $N$ denote any positive solution of (1.2)-(1.4). Let $q$ and $p$ denote solutions of the following:

$$
\left.\begin{array}{rl}
\frac{d q(t)}{d t} & =r(t) q(t)\left[1-\frac{q(t-n \tau)}{K(t)}\right] \\
q(s) & =\min _{x \in[0, \ell]} N(x, s), \quad s \in[-n \tau, 0]
\end{array}\right\} ;
$$

Then $N(x, t)$ satisfies

$$
q(t) \leqslant N(x, t) \leqslant p(t), \quad t \geqslant 0, \quad x \in[0, \ell] .
$$

Proof: We shall only show that

$$
N(x, t) \leqslant p(t), \quad \text { for } \quad t \geqslant 0, \quad x \in[0, \ell],
$$

since the proof of the other part is similar. Let $p_{\varepsilon}(t)$ denote the solution of

$$
\left.\begin{array}{rl}
\frac{d p_{\varepsilon}(t)}{d t} & =r(t) p_{\varepsilon}(t)\left[1-\frac{p_{\varepsilon}(t-n \tau)}{K(t)}\right] \\
p_{\varepsilon}(s) & =\max _{x \in[0, \ell]} N(x, s)+\varepsilon, \quad s \in[-n \tau, 0]
\end{array}\right\},
$$


where $\varepsilon$ is an arbitrary positive number. It is sufficient to show that

$$
N(x, t) \leqslant p_{e}(t), \quad t \geqslant 0, \quad x \in[0, \ell] .
$$

The conclusion (2.8) will then follow from (2.10) by the continuous dependence of $p_{e}$ on $\varepsilon$ and we can consider $\varepsilon \rightarrow 0$ so that

$$
N(x, t) \leqslant \lim _{\varepsilon \rightarrow 0} p_{e}(t)=p(t) .
$$

Suppose (2.10) does not hold; then there exists a point $\left(x_{0}, t_{0}\right), x_{0} \in[0, \ell], t_{0}>0$ such that

$$
p_{\varepsilon}\left(t_{0}\right)-N\left(x_{0}, t_{0}\right)<0 .
$$

We consider a function $M_{\mu}$ defined by

$$
M_{\mu}(x, t)=\left[p_{\varepsilon}(t)-N(x, t)\right] e^{-\mu t}, \quad(x, t) \in[0, \ell] \times\left[0, t_{0}\right]
$$

where $\mu$ is a positive number to be selected suitably. By continuity of $M_{\mu}$ on $[0, \ell] \times$ $\left[0, t_{0}\right]$, the function $M_{\mu}$ will have a negative minimum at some point say $\left(x_{*}, t_{*}\right) \in$ $[0, \ell] \times\left[0, t_{0}\right]$. From the fact that $M_{\mu}(x, 0)>0$, it will follow that $t_{*} \neq 0$. Thus $\left(x_{*}, t_{*}\right) \in[0, \ell] \times\left(0, t_{0}\right]$. If $\left(x_{*}, t_{*}\right) \in(0, \ell) \times\left(0, t_{0}\right]$ we then have

$$
\frac{\partial M_{\mu}\left(x_{*}, t_{*}\right)}{\partial t} \leqslant 0, \quad \frac{\partial^{2} M_{\mu}\left(x_{*}, t_{*}\right)}{\partial x^{2}} \geqslant 0 .
$$

But by a direct calculation one can derive that $M_{\mu}$ satisfies

$$
\begin{aligned}
\frac{\partial M_{\mu}(x, t)}{\partial t}= & \frac{\partial^{2} M_{\mu}(x, t)}{\partial x^{2}}+M_{\mu}(x, t)\left[r(t)-\mu-\frac{r(t)}{K(t)} p_{e}(t-n \tau)\right] \\
& -\frac{r(t)}{K(t)} N(x, t) e^{-\mu n \tau} M_{\mu}(x, t-n \tau) ; x \in(0, \ell), t \in\left(0, t_{0}\right]
\end{aligned}
$$

If we choose $\mu$ sufficiently large, then one can make the right side of (2.15) positive at $\left(x_{*}, t_{*}\right)$ while the left side of (2.15) stays nonpositive at $\left(x_{*}, t_{*}\right)$; but this is a contradiction. Thus $x_{*} \notin(0, \ell)$ and hence $x_{*}=0$ or $x_{*}=\ell$. Suppose that $M_{\mu}$ has a local negative minimum at $\left(0, t_{*}\right)$; we then have from the boundary condition that

$$
\frac{\partial M_{\mu}\left(0, t_{*}\right)}{\partial x}=0
$$

We can now proceed as in the case of $m_{\lambda}$ in the previous section to show that $M_{\mu}$ cannot have a negative minimum at the end points of the interval $(0, \ell)$. Thus $M_{\mu}$ cannot have a negative minimum on the closed set $[0, \ell] \times\left[0, t_{0}\right]$ for $t_{0}>0$ from which (2.10) follows. The validity of (2.8) follows from (2.11) and this completes the proof.

The following result has been established by Zhang and Gopalsamy [34] which we quote in full for the convenience of the reader. 
THEOREM 2.2. Assume that $r$ and $K$ are continuous strictly positive periodic functions with period $\tau>0$ such that

$$
n\langle r\rangle=\int_{0}^{n r} r(s) d s \leqslant \frac{3}{2} .
$$

Then the periodic logistic equation

$$
\frac{d x(t)}{d t}=r(t) x(t)\left[1-\frac{x(t-n \tau)}{K(t)}\right]
$$

has a unique positive periodic solution $y^{*}$ given by (2.2) or (2.3) and all other positive solutions of (2.17) satisfy

$$
\lim _{t \rightarrow \infty}\left|x(t)-y^{*}(t)\right|=0 .
$$

The next result is one of our main results and is established by an application of the conclusion of Theorem 2.2 .

Theorem 2.3. Let $D \in[0, \infty), \tau \in(0, \infty)$ and let $n$ be a positive integer. Let $r$ and $K$ denote strictly positive continuous periodic functions of period $\tau$. Then the Neumann periodic system (1.2) - (1.3) has a unique positive periodic solution $y^{*}$. Suppose further that $r$ and $\tau$ are such that

$$
n\langle r\rangle=\int_{0}^{n \tau} r(s) d s \leqslant \frac{3}{2}
$$

Then all the positive solutions of (1.2) - (1.4) converge to the unique periodic spatially homogeneous solution $y^{*}$ in the sense that

$$
\lim _{t \rightarrow \infty}\left|N(x, t)-y^{*}(t)\right|=0 \quad \text { uniformly in } x \in[0, \ell] .
$$

Proof: One can note immediately that $y^{*}$ is a periodic solution of $(1.2)-(1.3)$. Let $N$ denote any positive solution of (1.2) - (1.4). We have from Theorem 2.1 that

$$
q(t) \leqslant N(x, t) \leqslant p(t), \quad x \in[0, \ell], \quad t \geqslant 0
$$

which leads to

$$
q(t)-y^{*}(t) \leqslant N(x, t)-y^{*}(t) \leqslant p(t)-y^{*}(t), \quad x \in[0, \ell], \quad t \geqslant 0 .
$$

From (2.19) and Theorem 2.2, it follows that

$$
p(t)-y^{*}(t) \rightarrow 0 \text { and } q(t)-y^{*}(t) \rightarrow 0 \text { as } t \rightarrow \infty,
$$

and hence we derive from (2.22) that

$$
\lim _{t \rightarrow \infty}\left|N(x, t)-y^{*}(t)\right|=0, \quad x \in[0, \ell] .
$$

The uniqueness of $y^{*}(t)$ follows from its global attractivity as a solution of (1.2). This completes the proof. 


\section{Oscillations about the PERIOdIC SOLUTION}

In the previous section, we obtained sufficient conditions for all positive solutions of the periodic parabolic system (1.2) - (1.3) to converge to a spatially homogeneous and temporally periodic solution. One of the worthwhile questions regarding this convergence is the following: under what conditions is this convergence to periodicity oscillatory in the following sense?

Definition 3.1: A positive solution of (1.2) - (1.3) is said to be oscillatory about the positive periodic solution $y^{*}$ if for every $T>0$, there exists a point $\left(x_{0}, T_{0}\right) \in$ $(0, \ell) \times[T, \infty)$ such that $N\left(x_{0}, T_{0}\right)=y^{*}\left(T_{0}\right)$; a solution of $(1.2)-(1.3)$ is said to be nonoscillatory about $y^{*}$ if there exists a $T_{1}>0$ such that

$$
\left|N(x, t)-y^{*}(t)\right|>0 \text { for } x \in(0, \ell), \quad t>T_{1} .
$$

Although oscillations in delay and functional differential equations have been pursued extensively, oscillations of partial differential equations have not received much attention; oscillations of parabolic equations have been considered before by Kreith and Ladas [16] and Yoshida [33]. In the following we derive sufficient conditions for all positive solutions of $(1.2)-(1.3)$ to oscillate about the unique positive periodic solution $y^{*}$.

For convenience in the following we introduce a change of variable by the formula

$$
\log [1+u(x, t)]=\log [N(x, t)]-\log \left[y^{*}(t)\right]
$$

and find that $u$ is governed by

$$
\left.\begin{array}{c}
\frac{\partial u(x, t)}{\partial t}=D \frac{\partial^{2} u(x, t)}{\partial x^{2}}-\left(\frac{r(t) y^{*}(t)}{K(t)}\right)[1+u(x, t)] u(x, t-u \tau) \\
0<x<\ell, \quad t>0 \\
\frac{\partial u(0, t)}{\partial x}=\frac{\partial u(\ell, t)}{\partial x}=0, \quad t>-n \tau .
\end{array}\right\}
$$

One can note from (3.1) that oscillation of $N$ about $y^{*}$ is equivalent to that of $u$ about 0 or simply oscillation of $u$.

The oscillation of the lumped parameter system corresponding to (3.2), namely

$$
\frac{d v(t)}{d t}=-\left(\frac{r(t) y^{*}(t)}{K(t)}\right)[1+v(t)] v(t-n \tau)
$$

has been discussed by Zhang and Gopalsamy [34] and in particular they have shown that if

$$
\int_{t-n \tau}^{t} r(s) d s>\frac{1}{e}
$$


then every positive solution of (3.3) is oscillatory about $K$. The following result related to (3.3) not found in the work of Zhang and Gopalsamy [34] is of some interest and is complementary to the results of [3].

ThEOREM 3.1. Let $r$ and $K$ be positive continuous periodic functions of period $\tau>0$. If

$$
n \int_{0}^{\tau} r(s) d s>\frac{1}{e}
$$

then every positive solution of the periodic delay logistic equation

$$
\frac{d y(t)}{d t}=r(t) y(t)\left[1-\frac{y(t-n \tau)}{K(t)}\right]
$$

oscillates about the unique periodic solution $y^{*}$ of (3.6).

Proof: We let

$$
\log [1+v(t)]=\log [y(t)]-\log \left[y^{*}(t)\right]
$$

and find that $v$ is governed by (3.3). Assume that (3.5) holds. Suppose that $v$ is not oscillatory about zero; say $v$ is eventually positive; then the inequality

$$
\frac{d v(t)}{d t}<-\left(\frac{r(t) y^{*}(t)}{K(t)}\right) v(t-n \tau)
$$

has an eventually positive solution. We note that

$$
\frac{d y^{*}(t)}{d t}=r(t) y^{*}(t)\left(1-\frac{y^{*}(t)}{K(t)}\right)
$$

leads to

(3.10) $0=\log \frac{y^{*}(t)}{y^{*}(t-n \tau)}=\int_{t-n \tau}^{t} \frac{1}{y^{*}(t)} \frac{d y^{*}(t)}{d t} d t=\int_{t-n \tau}^{t} r(s) d s-\int_{t-n \tau}^{t} \frac{r(s) y^{*}(s)}{K(s)} d s$

and hence due to the periodicity of $r, y^{*}$ and $K$,

$$
\int_{t-n \tau}^{t} \frac{r(s) y^{*}(s)}{K(s)} d s=\int_{t-n \tau}^{t} r(s) d s=n \int_{0}^{r} r(s) d s>\frac{1}{e} .
$$

It is well known (see Koplatadze and Canturija [15]) that when (3.11) holds, all solutions of (3.8) are oscillatory and therefore $v$ cannot be eventually positive.

If $v$ is an eventually negative solution of (3.3) say

$$
v(t)<0 \text { for } t>T^{*},
$$


then we have from (3.3) that

$$
\frac{d v(t)}{d t} \leqslant-\left(\frac{r(t) y^{*}(t)}{K(t)}\right) v(t-n \tau) \text { for } t>T^{*}+n \tau
$$

and therefore an integration of both sides of (3.12) on $\left[T^{*}+n \tau, t\right]$ leads to

$$
v(t)-v\left(T^{*}+n \tau\right)+\int_{T^{*}+n \tau}^{t} \frac{r(s) y^{*}(s)}{K(s)} v(s-n \tau) d s \leqslant 0, \quad t>T^{*}+n \tau
$$

By the positive periodic and continuous nature of $r, y^{*}$ and $K$, it follows from (3.13) that $v$ is bounded and

$$
v \in L_{1}(0, \infty)
$$

But $v$ is uniformly continuous on $(0, \infty)$ due to the boundedness of $d v / d t$ on $(0, \infty)$ which is a consequence of the boundednes of $v$ on $(0, \infty)$. Then by Barbalatt's Lemma [2], it will follow that

$$
\lim _{t \rightarrow \infty} v(t)=0
$$

A consequence of (3.14) is that there exists a $T_{*}>T^{*}>0$ such that the eventually negative $v$ satisfies for arbitrary positive $\varepsilon$,

$$
v(t)>-\varepsilon \text { for } t>T_{*}
$$

and hence $1+v(t)>1-\varepsilon>0$ for $t>T_{*}$. Thus an eventually negative solution of (3.3) satisfies

$$
\frac{d v(t)}{d t}>-\left[\frac{r(t) y^{*}(t)}{K(t)}\right](1-\varepsilon) v(t-n \tau) \quad \text { for } \quad t>T_{*}+n \tau
$$

It is possible to choose an $\varepsilon>0$ such that for $t>T_{*}+n \tau$

$$
\int_{t-n \tau}^{t} \frac{r(s) y^{*}(s)}{K(s)} d s>\frac{1}{e} \Longrightarrow(1-\varepsilon) \int_{t-n \tau}^{t} \frac{r(s) y^{*}(s)}{K(s)} d s>\frac{1}{e}
$$

It is known (as before [15]) that when (3.16) holds, (3.15) and hence (3.3) cannot have an eventually negative solution. Thus when (3.5) holds, all positive solutions of (3.6) are oscillatory about the periodic solution $y^{*}$. This completes the proof.

The next result examines the qualitative and asymptotic behaviour of nonoscillatory solutions of (3.2) and is a preparation for our subsequent discussion (Theorem 3.3) of the oscillation of all solutions of the distributed parameter system (3.2). 
THEOREM 3.2. If $u(x, t)$ is a nonoscillatory solution of (3.2), then

$$
u(x, t) \rightarrow 0 \text { as } t \rightarrow \infty \text { uniformly in } x \in(0, \ell) .
$$

Proof: Suppose $u$ is an eventually positive solution of (3.2) (if $u$ is eventually negative, the details of proof will be similar). There exists a $T^{*}>0$ such that

$$
u(x, t)>0 \text { for } t>T^{*} \text { and } x \in(0, \ell) .
$$

Define $v$ as follows:

$$
v(t)=\int_{0}^{\ell} u(x, t) d x, \quad t>T^{*}+n \tau
$$

We have from (3.2) that

$$
\begin{aligned}
\frac{d v(t)}{d t} & =D \int_{0}^{\ell} \frac{\partial^{2} u(x, t)}{\partial x^{2}} d x-P(t) \int_{0}^{\ell}[1+u(x, t)] u(x, t-n \tau) d x \\
& =-P(t) \int_{0}^{\ell}[1+u(x, t)] u(x, t-n \tau) d x \\
& <0 \text { for } t>T+n \tau,
\end{aligned}
$$

where

$$
P(t)=\frac{r(t) y^{*}(t)}{K(t)}
$$

Since $v(t)>0$ for $t>T^{*}+n \tau$, it follows from (3.20) that $v(t) \rightarrow v^{*} \geqslant 0$ as $t \rightarrow \infty$. We derive from (3.19) that

$$
v(t)-v\left(T^{*}+n \tau\right)+\int_{T^{*}+n \tau}^{t} P(s)\left(\int_{0}^{\ell}[1+u(x, s)] u(x, s-n \tau) d x\right) d s=0 .
$$

Since $\lim _{t \rightarrow \infty} v(t)$ exists, we can conclude from (3.22) that

$$
\lim _{t \rightarrow \infty} \int_{T^{*}+n \tau}^{t} P(s) \int_{0}^{l}[1+u(x, s)] u(x, s-n \tau) d x d s \quad \text { exists }
$$

and therefore

$$
\lim _{t \rightarrow \infty} \int_{0}^{t} P(s) \int_{0}^{\ell}[1+u(x, s)] u(x, s-n \tau) d x d s \quad \text { exists. }
$$


For convenience we define $w$ as follows:

$$
w(t)=\frac{1}{\ell}\left[\int_{0}^{\ell} u(\xi, 0) d \xi+\int_{0}^{t} \int_{0}^{\ell} P(s) f(\xi, s) d \xi d s\right]
$$

where

$$
f(x, t)=-[1+u(x, t)] u(x, t-n \tau), \quad x \in(0, \ell)
$$

It can be found from (3.23) - (3.25) that there exists a number $w^{*} \in R$ such that

$$
\lim _{t \rightarrow \infty} w(t)=w^{*}
$$

Let $G(x, t ; \xi, s)$ denote the Green's function associated with the Neumann boundary value problem (3.2). Then for any solution $u(x, t)$ of (3.2) we can write (for more details see Kahane $[11,12]$ )

$$
u(x, t)=\left\{\begin{array}{l}
\int_{0}^{\ell} G(x, t ; \xi, 0) u(\xi, 0) d \xi+\int_{0}^{t} \int_{0}^{\ell} G(x, t ; \xi, s) P(s) f(\xi, s) d \xi d s, \quad t>0 \\
\phi(x, t), \quad t \in[-n \tau, 0], \quad x \in[0, \ell]
\end{array}\right.
$$

Using (3.24) we then have

$$
\begin{aligned}
u(x, t)-w(t)= & \int_{0}^{\ell}\left[G(x, t ; \xi, 0)-\frac{1}{\ell}\right] u(\xi, 0) d \xi \\
& +\int_{0}^{t} \int_{0}^{\ell}\left[G(x, t ; \xi, s)-\frac{1}{\ell}\right] P(s) f(\xi, s) d \xi
\end{aligned}
$$

for $x \in(0, \ell), t>0$. It is known that the Green's function satisfies (see Kahane [11, 12])

$$
\left.\begin{array}{l}
\left|G(x, t ; \xi, s)-\frac{1}{\ell}\right| \leqslant c_{1} e^{-c_{2}(t-s)}, \quad t-s \geqslant 1, \quad x, \xi \in(0, \ell) \\
\left|G(x, t ; \xi, s)-\frac{1}{\ell}\right| \leqslant\left(\frac{1}{t-s}\right)^{1 / 2}, \quad t-s>0, \quad x, \xi \in(0, \ell)
\end{array}\right\}
$$

where $c_{1}, c_{2}$ and $c$ are positive constants. It is easy to derive from the first of (3.28) that

$$
\lim _{t \rightarrow \infty} \int_{0}^{\ell}\left|G(x, t ; \xi, 0)-\frac{1}{\ell}\right||u(\xi, 0)| d \xi=0
$$


and the convergence in (3.29) is uniform in $x \in[0, \ell]$. Before we consider the limiting behaviour as $t \rightarrow \infty$ of the second integral on the right side of (3.27), we shall show that

$$
\int_{0}^{\ell}|f(x, t)| d x \rightarrow 0 \quad \text { as } \quad t \rightarrow \infty
$$

From (3.19) and the eventual positivity of $u$, we have

$$
\begin{aligned}
\frac{d v(t)}{d t} & =-P(t) \int_{0}^{\ell}[1+u(x, t)] u(x, t-n \tau) d x \\
& <-P(t) \int_{0}^{\ell} u(x, t-n \tau) d x<0 .
\end{aligned}
$$

It will follow from (3.31) and (3.32), due to the bounded positive periodic nature of $P$, that

$$
\left.\begin{array}{r}
\int_{0}^{\ell}[1+u(x, t)] u(x, t-n \tau) d x \in L_{1}(0, \infty) \\
\int_{0}^{\ell} u(x, t) d x \in L_{1}(0, \infty) .
\end{array}\right\}
$$

Since $u>0$ eventually we also have from (3.33) that

$$
\int_{0}^{\ell} u(x, t) u(x, t-n \tau) d x \in L_{1}(0, \infty)
$$

We now let

$$
Q(t)=\int_{0}^{\ell} u^{2}(x, t) d x ; \quad t>0
$$

and we derive that

$$
\begin{aligned}
\frac{d Q(t)}{d t} & =2 \int_{0}^{\ell} u(x, t) \frac{\partial u(x, t)}{\partial t} d x \\
& =2 \int_{0}^{\ell} u(x, t)\left[D \frac{\partial^{2} u(x, t)}{\partial x^{2}}+P(t) f(x, t)\right] d x \\
& =-2 D \int_{0}^{\ell}\left(\frac{\partial u(x, t)}{\partial x}\right)^{2} d x+2 \int_{0}^{\ell} u(x, t) P(t) f(x, t) d x<0
\end{aligned}
$$


We have from (3.36) and (3.25) that

$$
\left.\begin{array}{r}
\int_{0}^{\ell}\left(\frac{\partial u(x, t)}{\partial x}\right)^{2} d x \in L_{1}(0, \infty) \\
\int_{0}^{\ell} u(x, t) P(t)[1+u(x, t)] u(x, t-n \tau) d x \in L_{1}(0, \infty) .
\end{array}\right\}
$$

If $F$ is defined by

$$
F(t)=\int_{0}^{\ell}[1+u(x, t)] u(x, t-n \tau) d x, \quad t>n \tau
$$

then from (3.33), we conclude that $F \in L_{1}(n \tau, \infty)$. We proceed to establish that

$$
\left|\frac{d F(t)}{d t}\right| \in L_{1}(n \tau, \infty)
$$

By direct calculation from (3.38), (3.2) and (3.25),

$$
\begin{aligned}
\frac{d F(t)}{d t}= & \int_{0}^{\ell} u(x, t-n \tau)\left\{D \frac{\partial^{2} u(x, t)}{\partial x^{2}}+P(t) f(x, t)\right\} d x \\
& +\int_{0}^{\ell}[1+u(x, t)]\left\{D \frac{\partial^{2} u(x, t-n \tau)}{\partial x^{2}}+P(t-n \tau) f(x, t-n \tau)\right\} d x \\
= & -D \int_{0}^{\ell} \frac{\partial u(x, t)}{\partial x} \frac{\partial u(x, t-n \tau)}{\partial x} d x+\int_{0}^{\ell} u(x, t-n \tau) P(t) f(x, t) d x \\
& -D \int_{0}^{\ell} \frac{\partial u(x, t)}{\partial x} \frac{\partial u(x, t-n \tau)}{\partial x} d x \\
& +\int_{0}^{\ell}[1+u(x, t)] P(t-n \tau) f(x, t-n \tau) d x \\
= & -2 D \int_{0}^{\ell} \frac{\partial u(x, t)}{\partial x} \frac{\partial u(x, t-n \tau)}{\partial x} d x \\
& +\int_{0}^{\ell}[1+u(x, t)] P(t-n \tau) f(x, t-n \tau) d x \\
& +\int_{0}^{\ell} u(x, t-n \tau) P(t) f(x, t) d x
\end{aligned}
$$


We obtain from (3.42) that for $t>T>n \tau$,

$$
\begin{gathered}
F(t)+\int_{T}^{t}\left[\int_{0}^{\ell}[1+u(x, s)] P(s-n \tau)|f(x, s-n \tau)| d x\right. \\
\left.\quad+\int_{0}^{\ell} u(x, s-n \tau) P(s)|f(x, s)| d x\right] d s \\
\leqslant F(T)-2 D \int_{T}^{t} \int_{0}^{\ell} \frac{\partial u(x, s)}{\partial x} \frac{\partial u(x, s-n \tau)}{\partial x} d x d s \\
\leqslant F(T)+D \int_{0}^{t}\left[\int_{0}^{\ell}\left\{\left(\frac{\partial u(x, s)}{\partial x}\right)^{2}+\left(\frac{\partial u(x, s-n \tau)}{\partial x}\right)^{2}\right\} d x\right] d s \\
<\infty \text { on using (3.37). }
\end{gathered}
$$

It follows from (3.43) and the periodicity of $P$ that

$$
\left.\begin{array}{r}
\int_{0}^{\ell}[1+u(x, t)] P(t-n \tau)|f(x, t-n \tau)| d x \in L_{1}(T, \infty) \\
\int_{0}^{\ell} u(x, t-n \tau) P(t)|f(x, t)| d x \in L_{1}(T, \infty) .
\end{array}\right\}
$$

We can conclude from the first of (3.37) and (3.42) - (3.44) that (3.39) holds. Thus both $F$ and $d F / d t$ belong to $L_{1}(T, \infty)$. By Lemma 1 of the Appendix it will follow that

$$
F(t)=\int_{0}^{\ell}[1+u(x, t)] u(x, t-n \tau) d x=\int_{0}^{\ell}|f(x, t)| d x \rightarrow 0 \text { as } t \rightarrow \infty .
$$

To investigate the asymptotic behaviour of the second integral on the right side of (3.27), we define $z(t)$ in the form:

$$
z(t)=\int_{0}^{t} \int_{0}^{\ell}\left[G(x, t ; \xi, s)-\frac{1}{\ell}\right] P(s) f(\xi, s) d \xi d s
$$

and proceed to estimate $z(t)$ as follows:

$$
\begin{aligned}
|z(t)| \leqslant & \int_{0}^{T} \int_{0}^{\ell}\left|G(x, t ; \xi, s)-\frac{1}{\ell}\right| P(s)|f(\xi, s)| d \xi d s \\
& +\int_{0}^{t-1} \int_{0}^{\ell}\left|G(x, t ; \xi, s)-\frac{1}{\ell}\right| P(s)|f(\xi, s)| d \xi d s \\
& +\int_{t-1}^{t} \int_{0}^{\ell}\left|G(x, t ; \xi, s)-\frac{1}{\ell}\right| P(s)|f(\xi, s)| d \xi d s .
\end{aligned}
$$


Each of the terms on the right side of (3.47) is estimated in the following way:

$$
\begin{aligned}
\int_{t-1}^{t} \int_{0}^{\ell} \mid & G(x, t ; \xi, s)-\frac{1}{\ell}|P(s)| f(\xi, s) \mid d \xi d s \\
& \leqslant \int_{t-1}^{t} \int_{0}^{\ell} c\left(\frac{1}{t-s}\right)^{1 / 2} P(s)|f(\xi, s)| d \xi d s \\
& \leqslant c P^{*} \int_{0}^{\ell}\left(\frac{1}{\alpha}\right)^{\frac{1}{2}}\left(\int_{0}^{\ell}|f(\xi, t-\alpha)| d \xi\right) d \alpha \\
& \rightarrow 0 \text { as } t \rightarrow \infty \text { on using }(3.45)
\end{aligned}
$$

where

$$
P^{*}=\max _{t \in[0, \tau]}\left\{\frac{r(t) y^{*}(t)}{K(t)}\right\}
$$

For an arbitrary $\varepsilon>0$,

$$
\begin{gathered}
\int_{T}^{t-1} \int_{0}^{\ell}\left|G(x, t ; \xi, s)-\frac{1}{\ell}\right| P(s)|f(\xi, s)| d \xi d s \\
\leqslant c_{1} P^{*} \int_{T}^{t-1} \int_{0}^{\ell} e^{-c_{2}(t-s)}|f(\xi, s)| d \xi d s \\
\leqslant c_{1} P^{*} \int_{T}^{t} \int_{0}^{\ell}|f(\xi, s)| d \xi d s \\
\quad<\varepsilon P^{*} \quad \text { if } T \text { is sufficiently large. } \\
\int_{0}^{T} \int_{0}^{\ell}\left|G(x, t ; \xi, s)-\frac{1}{\ell}\right| P(s)|f(\xi, s)| d \xi d s \\
\leqslant c_{1} P^{*} \int_{0}^{T} \int_{0}^{\ell} e^{-c_{2}(t-s)}|f(\xi, s)| d \xi d s \\
\leqslant \frac{c_{1} P^{*} \int_{0}^{t} e^{c_{2} \cdot}\left(\int_{0}^{\ell}|f(\xi, s)| d \xi\right) d s}{e^{c_{2} t}} \\
\rightarrow 0 \quad \text { as } t \rightarrow \infty
\end{gathered}
$$

by (3.45) and L'Hopital's rule. Thus each of the three integrals on the right side of (3.47) can be made arbitrarily small for large enough $t$ by a suitable choice of $T$ and this leads to

$$
\lim _{t \rightarrow \infty} z(t)=\lim _{t \rightarrow \infty} \int_{0}^{t} \int_{0}^{\ell}\left[G(x, t ; \xi, s)-\frac{1}{\ell}\right] P(s) f(\xi, s) d \xi d s=0 .
$$

It follows from (3.27) - (3.51) that

$$
u(x, t) \rightarrow w(t) \text { as } t \rightarrow \infty \text {, uniformly in } x \in(0, \ell) .
$$


But we know that

$$
w(t) \rightarrow w^{*} \text { as } t \rightarrow \infty
$$

and therefore

$$
u(x, t) \rightarrow w^{*} \text { as } t \rightarrow \infty \text { uniformly in } x \in(0, \ell) .
$$

From (3.24), we have

$$
\frac{d w(t)}{d t}=-\frac{P(t)}{\ell} \cdot \int_{0}^{\ell}[1+u(x, t)] u(x, t-n \tau) d x
$$

which together with (3.23) leads to

$$
\frac{d w(t)}{d t} \in L_{1}[0, \infty)
$$

On the other hand we have from (3.53),

$$
\lim _{t \rightarrow \infty} \int_{0}^{\ell}[1+u(x, t)] u(x, t-n \tau) d x=\ell\left(1+w^{*}\right) w^{*} .
$$

We now claim $w^{*}=0$. If this is not the case then we have from the eventual positivity of $u$ that $w^{*}>0$. As a consequence there exists $t^{*}>0$ such that

$$
u(x, t)>\frac{w^{*}}{2} \quad \text { for } \quad x \in(0, \ell), \quad t>t^{*} .
$$

We will then have from (3.54) that for $t>t^{*}+n \tau$,

$$
\begin{aligned}
w(t)-w\left(t^{*}\right) & =-\frac{1}{\ell} \int_{t^{*}}^{t} \int_{0}^{\ell} P(s)[1+u(x, s)] u(x, s-n \tau) d s d x \\
& <-P_{*}\left(1+\frac{w^{*}}{2}\right) \frac{w^{*}}{2}\left(t-t^{*}\right)
\end{aligned}
$$

where

$$
\min _{\cdot \in[0, \tau]} P(s)=P_{*}>0 \text {; }
$$

the positivity of $P_{*}$ is a consequence of the periodicity, continuity and the positivity of $r, y^{*}$ and $K$. An implication of (3.55) is that

$$
\boldsymbol{w}(t) \rightarrow-\infty \quad \text { as } \quad t \rightarrow \infty
$$


and this contradicts the existence of $w^{*}>0$. Thus $w^{*}=0$ and therefore we have from the above that

$$
u(x, t) \rightarrow w(t) \rightarrow w^{*}=0 \quad \text { as } \quad t \rightarrow \infty .
$$

The proof is complete.

The next result provides sufficient conditions for all positive solutions of (1.2) (1.3) to oscillate about the unique positive periodic solution $y^{*}$ of $(1.2)-(1.3)$. The conclusion of the result of Theorem 3.2 will be used in the proof of the next result.

Theorem 3.3. Assume that

$$
\int_{0}^{n \tau} r(s) d s=\int_{t-n \tau}^{t} r(s) d s>\frac{1}{e}
$$

or equivalently

$$
\int_{0}^{\tau} r(s) d s=\int_{t-\tau}^{t} r(s) d s>\frac{1}{n e} .
$$

Then all positive solutions of (1.2) - (1.3) are oscillatory about the unique positive periodic solution $y^{*}$ of (1.2) - (1.3).

Proof: It can be found from (3.1) that it is sufficient to consider the oscillation of solutions of (3.2). Our strategy of proof is to assume that (3.2) has a nonoscillatory solution say $u(x, t)$ and then show that this leads to a contradiction. First we suppose that $u(x, t)$ is eventually positive, that is there is $T^{*}>0$ such that

$$
u(x, t)>0, \text { for } t>T^{*}, \quad x \in(0, \ell) .
$$

We define

$$
v(t)=\int_{0}^{\ell} u(x, t) d x \text { for } t>T^{*}
$$

and note that $v$ is eventually positive say for $t>T^{*}$. Then from (3.19)

$$
\begin{aligned}
\frac{d v(t)}{d t} & =-P(t) \int_{0}^{\ell}[1+u(x, t)] u(x, t-n \tau) d x \\
& \leqslant-P(t) \int_{0}^{\ell} u(x, t-n \tau) d x \\
& =-P(t) v(t-n \tau) \text { for } t>T^{*}+n \tau .
\end{aligned}
$$

It follows that the differential inequality (3.57) has an eventually positive solution. From (3.10), we have

$$
\int_{t-n \tau}^{t} P(s) d s=\int_{0}^{n \tau} r(s) d s=n \int_{0}^{\tau} r(s) d s>\frac{1}{e} .
$$


It is known (see [15]) that (3.57) cannot have an eventually positive solution when (3.58) holds.

Suppose now that $u(x, t)$ is eventually negative; then there exists $T_{1}>0$ such that

$$
1+u(x, t)<1, \quad v(t)<0, \quad \text { for } \quad t>T_{1} .
$$

By Theorem 3.2, we know

$$
\lim _{t \rightarrow \infty} u(x, t)=0 \quad \text { uniformly in } \quad x \in(0, \ell)
$$

So for any given $\varepsilon>0$ there is $T_{2}>T_{1}$ such that

$$
1+u(x, t)>1-\varepsilon \quad \text { for } \quad t>T_{2},
$$

and therefore

$$
\frac{d v(t)}{d t} \geqslant-P(t)(1-\varepsilon) v(t-n \tau) \quad \text { for } \quad t>T_{2}+n \tau
$$

Since $\varepsilon$ is arbitrary, it is possible to have from (3.56) and (3.58) that

$$
(1-\varepsilon) \int_{0}^{n \tau} P(s) d s>\frac{1}{e}
$$

for some $\varepsilon>0$. It is again known to be impossible for (3.59) to have an eventually negative solution when (3.60) holds (see [15]). The result follows from these contradictions.

We conclude with the following few remarks: the existence of a globally attracting positive periodic solution has certain ecobiological (ecological and biological) consequences; oscillations of all solutions due to the time delay can enhance the development of "fitness" of the species modelled by (1.2). These aspects have been discussed in the absence of spatial heterogeneity by Gopalsamy, He and Wen [7]. As special cases, sufficient conditions for the global asymptotic stability of the positive equilibrium of the autonomous analogue of (1.2) - (1.3) where $r$ and $K$ are positive constants are implicit in Theorem 2.3; and similarly Theorem 3.3 provides sufficient conditions for the oscillation of positive solutions of (1.2) - (1.3) about the positive equilibrium $K$ of the autonomous analogue of (1.2) - (1.3). These results related to the autonomous analogue of (1.2) - (1.3) are also new to the literature on reaction diffusion equations with time delays. 


\section{ApPEndix}

LEMma 1. Let $f:[0, \infty) \mapsto[0, \infty)$ be continuously differentiable on $(0, \infty)$ such that

Then

$$
f \in L_{1}(0, \infty) \quad \text { and } \quad \frac{d f}{d t} \in L_{1}[0, \infty)
$$

$$
\lim _{t \rightarrow \infty} f(t)=0 \text {. }
$$

Proof: Since $d f / d t \in L_{1}[0, \infty)$, for every $\varepsilon>0$ there exists a positive number $T>0$ such that for $t_{1}, t_{2}$ and $t_{2}>t_{1}>T$

$$
\left|\int_{t_{1}}^{\infty} \frac{d f(t)}{d t} d t\right|<\frac{\varepsilon}{2} ;\left|\int_{t_{2}}^{\infty} \frac{d f(t)}{d t} d t\right|<\frac{\varepsilon}{2} .
$$

We have from

$$
\begin{aligned}
\left|f\left(t_{2}\right)-f\left(t_{1}\right)\right| & =\left|\int_{t_{1}}^{t_{2}} \frac{d f(t)}{d t} d t\right| \\
& =\left|\int_{t_{1}}^{\infty} \frac{d f(t)}{d t} d t-\int_{t_{2}}^{\infty} \frac{d f(t)}{d t} d t\right| \\
& \leqslant\left|\int_{t_{1}}^{\infty} \frac{d f(t)}{d t} d t\right|+\left|\int_{t_{2}}^{\infty} \frac{d f(t)}{d t} d t\right| \\
& <\frac{\varepsilon}{2}+\frac{\varepsilon}{2} .
\end{aligned}
$$

It follows that $\lim _{t \rightarrow \infty} f(t)$ exists; this together with the facts

$$
f(t)>0, \quad f \in L_{1}[0, \infty)
$$

will imply that

$$
\lim _{t \rightarrow \infty} f(t)=0 \text {. }
$$

\section{References}

[1] H. Amann, 'Periodic solutions of semilinear parabolic equations', in Nonlinear analysis, Editors L. Cesari, R. Kannan and H.F. Weinberger, pp. 1-29 (Academic Press, New York, 1978).

[2] I. Barbalatt, 'Systemes d'equations differentielle d'oscillations nonlineaires', Rev. Roumaine Math. Pures Appl. 4 (1959), 267-270.

[3] N.F. Britton, Reaction-diffusion equations and their applications to biology (Academic Press, London, 1986). 
[4] D.S. Cohen and S. Rosenblatt, 'Multispecies interactions with hereditary effects and spatial diffusion', J. Math. Biol. 7 (1979), 231-241.

[5] B.D. Coleman, 'Nonautonomous logistic equations as model of the adjustment of populations to environmental change', Math. Biosci. 45 (1979), 159-173.

[6] B.D. Coleman, 'On optimal intrinsic growth rates for populations in periodically changing environment', J. Math. Biol. 12 (1981), 343-354.

[7] K. Gopalsamy, Xue-zhong He and Lizhi Wen, 'Global attractivity and oscillations in a periodic logistic integrodifferential equation', Houston J. Math. 17 (1991), 157-177.

[8] D. Green and H.W. Stech, 'Diffusion and hereditary effects in a class of population models', in Differential equations and applications in ecology, epidemics and population problems, Editors S. Busenberg and K.L. Cooke, pp. 19-28 (Academic Press, New York, 1981).

[9] G.E. Hutchinson, 'Circular causal systems in ecology', Ann. New York Acad. Sci. 50 (1948), 221-246.

[10] G.S. Jones, 'On the nonlinear difference differential equation $f^{\prime}(x)=-\alpha f(x-1)[1+$ $f(x)$ ]', J. Math. Anal. Appl. 4 (1962), 440-469.

[11] C.S. Kahane, 'On the asymptotic behaviour of solutions of parabolic equations', Czechoslovak Math. J. 33 (1983), 262-285.

[12] C.S. Kahane, 'On the asymptotic behaviour of solutions of parabolic equations under homogeneous Neumann boundary conditions', Funkcial. Ekvac. 32 (1989), 191-213.

[13] S. Kakutani and L. Markus, 'On the nonlinear difference differential equation $y^{\prime}(t)=$ $[A-B y(t-\tau)] y(t)$ ', in Contribution to the theory of nonlinear oscillations (Princeton University Press, 1958).

[14] Ju. S. Kolesov, 'Periodic solutions of quasilinear parabolic equations of second order', Trans. Moscow Math. Soc. 21 (1970), 114-146.

[15] R.G. Koplatadze and T.A. Canturija, 'On oscillatory and monotone solutions of first order differential equations with deviating arguments', Differentsial'nye Uravneniya 18 (1982), 1463-1465.

[16] K. Kreith and G. Ladas, 'Allowable delays for positive diffusion processes', Hiroshima Math. J. 15 (1985), 437-443.

[17] A.W. Leung, Systems of nonlinear partial differential equations (Kluwer Academic Publishers, Dordrecht, 1989).

[18] J. Lin and P.B. Khan, 'Phase and amplitude in delay-diffusion population models', $J$. Math. Biol. 13 (1982), 383-393.

[19] S. Luckhaus, 'Global boundedness for a delay differential equation', Trans. Amer. Math. Soc. 294 (1986), 767-774.

[20] M.C. Memory, 'Bifurcation and asymptotic behaviour of solutions of a delay differential equation with diffusion', SIAM J. Appl. Math. 20 (1989), 533-546.

[21] Y. Morita, 'Instability of spatially homogeneous periodic solutions to delay diffusion equations', in Lecture Notes Numer. Appl. Anal. 6 (1983), 107-124. (Recent Topics in Nonlinear PDE, Hiroshima, 1984).

[22] J.D. Murray, 'Spatial structures in predator-prey communities - a nonlinear time delay diffusion model', Math. Biosci. 30 (1976), 73-85. 
[23] A. Okubo, Diffusion and ecological problems: Mathematical models (Springer-Verlag, Berlin, 1980).

[24] M.H. Protter and H.F. Weinberger, Maximum principles in differential equations (Prentice-Hall Englewood Cliffs, New Jersey, 1967).

[25] R. Redlinger, 'On Volterra's population equation with diffusion', SIAM J. Math. Anal. 36 (1985), 135-142.

[26] A. Schiaffino, 'On a diffusion Volterra equation', Nonlinear Anal. 3 (1979), 595-600.

[27] A. Tesei, 'Stability properties of partial Volterra integrodifferential equations', Ann. Mat. Pura Appl. 126 (1980), 103-115.

[28] O. Vejvoda, Partial differential equations: Time-periodic solutions (Martinus Nijhoff Publishers, The Hague, 1982).

[29] E.M. Wright, 'A nonlinear difference differential equation', J. Reine and Angew. Math. 194 (1955), 66-87.

[30] Y. Yamada, 'On a certain class of semilinear Volterra diffusion equations', J. Math. Anal. Appl. 88 (1982), 433-451.

[31] K. Yoshida, 'The Hopf bifurcation and its stability for semilinear diffusion equation with time delay arising in ecology', Hiroshima Math. J. 12 (1982), 321-348.

[32] K. Yoshida and K. Khishimoto, 'Effect of two delays on partially functional differential equations', Kuramoto J. Sci. (Math.) 15 (1983), 91-109.

[33] K. Yoshida, 'Oscillation of nonlinear parabolic equations with functional arguments', $\mathrm{Hi}$ roshima Math. J. 16 (1986), 305-314.

[34] B.G. Zhang and K. Gopalsamy, 'Global attractivity and oscillations in a periodic delay-logistic equation', J. Math. Anal. Appl. 150 (1990), 274-283.

School of Information Science and Technology Flinders University

G.P.O. Box 2100

Adelaide SA 5001

Australia
Department of Mathematics

South China Normal University

Guangzhou

Peoples Republic of China 